
\title{
25 Research Soure \\ Anti-fibrotic effect of human fetal skin-derived stem cell secretomeon the liver fibrosis
}

\section{Xia Yao}

Affiliated Hospital of Changchun University of Chinese Medicine

Jing Wang

Affiliated Hospital of Changchun University of Chinese Medicine

Jiajing Zhu

China-Japan Union Hospital of Jilin University

Xiaoli Rong ( $\nabla$ rongxl16@mails.jlu.edu.cn )

Johns Hopkins University

\section{Research}

Keywords: hFSSC, secretome, liver fibrosis, TGF- $\beta /$ Smad

Posted Date: June 8th, 2020

DOl: https://doi.org/10.21203/rs.3.rs-19079/v3

License: (9) This work is licensed under a Creative Commons Attribution 4.0 International License. Read Full License 


\section{Abstract}

Background: Liver fibrosis resulting from a chronic liver injury is one of the significant causes of mortality. Stem cells-secreted secretome has been evaluated for overcoming the limitations of cell-based therapy in hepatic disease while maintaining its advantages over the current therapies.

Methods: In this study, we investigated the effect of human fetal skin-derived stem cells (hFSSCs) secretome in the treatment of liver fibrosis. To determine the therapeutic potential of the hFSSCs secretome in liver fibrosis, we established the $\mathrm{CCl}_{4}$-induced liver fibrosis rat model, and we administered hFSSCs secretome in vivo. Moreover, we investigated the anti-fibrotic mechanism of hFSSCs secretome in hepatic stellate cells (HSCs).

Results: Our results showed that hFSSCs secretome effectively reduced collagen content in the liver, and improved the liver function and promoted liver regeneration. Interestingly, we also found that hFSSCs secretome reduced liver fibrosis through suppressing the epithelial-mesenchymal transition (EMT) process. In addition, we found that hFSSC secretome inhibited the TGF- $\beta 1$, Smad2, Smad3, and Collagen I expression, while we observed, increased Smad7 expression.

Conclusions: In conclusion, our results suggest that hFSSCs secretome treatment could reduce $\mathrm{CCl}_{4}$ induced liver fibrosis via regulating the TGF- $\beta$ /Smad signal pathway.

\section{Introduction}

Liver fibrosis is a wound-healing response generated against chronic or iterative liver injury[1]. Recent evidence suggests that stem cell-based liver fibrosis treatment can be mediated through paracrine effects[2,3]. The exclusive use of stem cell-secreted secretome has been evaluated for overcoming the limitations and challenges of cell-based therapy while maintaining its advantages to their parent cells[4]. It included those extracellular vesicles, and other soluble proteins or biologically active molecules. In addition, previous studies have indicated that human bone marrow mesenchymal stem cells-derived exosomes and other stem cells-derived secretome can reduced liver fibrosis[5, 6].

Previous studies have indicated that the features of fetal tissue cells facilitate engraftment in vivo and may provide preferred effects against diseases challenging to treat[7]. Since 1928, hundreds of clinical trials using various types of fetal transplants have been performed worldwide[8-10]. Moreover, recent studies have been demonstrated that human fetal stem cells have a significant growth promoting potential, which benefited to tissue regeneration and cell therapy[11, 12]. In comparison to other mesenchymal stem cells (MSCs), fetal stem cells (FSCs) are easier to culture and more readily proliferate, and less likely to be rejected by transplant recipients, as these cells are less antigenic[8]. Although there remain still ethical and social issues concerning the clinical use of fetal tissue, fetal stem cell secretome transplantation may overcome these problems and have more perspectives on hepatic disease treatment. 
In our previous study, we have successfully isolated and characterized hFSSCs therapeutic quality control[13]. Interestingly, we found that hFSSC secretome has a great ability to control and balance the collagen formation in skin tissue[13]. Therefore, we hypothesis that hFSSC has a potential to reduce collagen formation in liver fibrosis, whereas liver fibrosis is caused by overabundance of Collagen. We further analyzed the effects of hFSSC secretome on live fibrosis in vitro, and the investigated the mechanism of hFSSC secretome and TGF- $\beta$ /Smad pathway involvement on anti-fibrosis. Our results provide the first evidence that hFSSCs secretome effectively reduces liver fibrosis through the TGF$\beta /$ Smad pathway. We believe that the hFSSCs secretome as an acellular regenerative therapy and approaches can provide great potential for the treatment of liver fibrosis.

\section{Materials And Methods}

\section{Cell culture}

hFSSCs and human umbilical cord mesenchymal stem cells (hUCMSCs) were provided and isolated by following our previous study[13]. HSCs were purchased from the Chinese Academy of Medical Sciences, China. In brief, hFSSCs, hUCMSCs, and HSCs were cultured in DMEM (Gibco, Grand island, U.S.) supplemented with $500 \mathrm{U} / \mathrm{ml}$ penicillin and $500 \mu \mathrm{g} / \mathrm{ml}$ streptomycin (Invitrogen, Shanghai, China), and $10 \%$ FBS (Gibco, Grand island, U.S.) at $37^{\circ} \mathrm{C}$, with saturated humidity and $5 \% \mathrm{CO}_{2}$. hFSSCs and hUCMSCs at the P5 were used for this study, and hFSSCs and hUCMSCs) secretome was collected as reported in our previous study[13]. Briefly, cells were cultured and reached $70 \sim 80 \%$ confluence, placed in serum-free medium (SFM; Invitrogen, Shanghai, China), and incubated in $5 \% \mathrm{CO}_{2}$ in a humidified condition. After cultured $24 \mathrm{~h}$, the conditioned medium (CM) was collected and centrifuged to purify for 10 min at $4{ }^{\circ} \mathrm{C}$, 4000g. Next, $10 \mathrm{ml}$ conditioned medium was re-centrifuged with Amicon Ultra Centrifugal Filters (Millipore Corp, Billerica, MA, USA) at $4{ }^{\circ} \mathrm{C}, 4000 \mathrm{~g}, 2 \mathrm{~h}$. At last, 300 500 $\mu \mathrm{l}$ supernatant solution was collected as cell-free secretome each time. The protein content was measured using the BCA kit (Thermo Scientific, Rockford, IL, USA) according to the manufacturer's instruction.

\section{$\mathrm{CCl}_{4}$-induced liver fibrosis in rats}

Liver fibrosis was induced in Sprague Dawley (SD) rats (8-week old, female, 200g). All protocols and procedures were approved by the Animal Experiment Ethics Committee of Changchun University of Traditional Chinese Medicine (Approval NO. XW201903167). Detailed procedures for the $\mathrm{CCl}_{4}$-induced liver fibrosis rat model were described in our previous published studies[6]. Briefly, rats were administered with an intraperitoneal injection of $30 \% \mathrm{CCl}_{4}, 3 \mathrm{ml} / \mathrm{kg}$ body weight twice weekly in olive oil. After eight weeks, $\mathrm{CCl}_{4}$ treated rats were randomly assigned into three groups $(\mathrm{n}=10$ rats, tail vein injection $(1 \mathrm{ml}) /$ weekly): PBS group (receive sterile PBS); hUCMSC secretome group (receive $250 \mu \mathrm{g}$ of hUCMSC secretome); hFSSC secretome group (receive hFSSC secretome of $250 \mu \mathrm{g}$ ). After 4 weeks, rats were sacrificed, liver tissue and serum were collected. Livers were divided into two parts. one part were preserved in $10 \%$ formalin (Histology studies) and other part flash frozen and kept in at $-80{ }^{\circ} \mathrm{C}$ (biochemical studies). 


\section{Histopathological analysis}

\section{DAB Staining:}

Liver tissues were processed for paraffin embedding by slicing into $4 \mu \mathrm{m}$ sections. Liver sections were stained with hematoxylin \& eosin (H\&E) and Masson and Sirius red according to standard protocols. Immunohistochemistry (IHC) was stained with the Kit (Maixin KIT-9710, Fuzhou, China) in accordance with the manufacturer's instructions. In brief, the liver sections were deparaffinized, rehydrated, and incubated in a $100{ }^{\circ} \mathrm{C}$ water bath for 15 minutes. Then, the slide was incubated with $3 \% \mathrm{H}_{2} \mathrm{O}_{2}$ for 15 minutes, and blocked with $10 \%$ normal goat serum for $1 \mathrm{~h}$ at $37^{\circ} \mathrm{C}$. Following with the incubation of primary antibody against PCNA (ab15497, 1:500 dilution, Abcam, Cambridge, UK), a-SMA (ab5694, 1:500 dilution, Abcam, Cambridge, UK), and HNF-4a(ab219610, 1:500 dilution, Abcam, Cambridge, UK) overnight at $4{ }^{\circ} \mathrm{C}$. Next, slides were incubated with biotinylated goat-anti-rabbit IgG antibody. Add diaminobenzidine solution for 15 minutes at $37^{\circ} \mathrm{C}$, then incubated with avidin peroxidase reagent, and hematoxylin for counterstaining. Lastly, slides were photographed using an optical microscope (Olympus, Tokyo Metropolitan, Japan). We selected the liver section fields randomly (used 10 random fields) to analyze the intensity liver fibrosis. We used 10 sections in total ( $\mathrm{n}=10$ rats) for quantification of IHC results. The percentage of collagen stained area was calculated via Image-Pro Plus software.

\section{Immunofluorescence (IF) staining}

When HSCs reached $60 \sim 70 \%$ confluence on 24-well plates, they were cultured with with either PBS, hUCMSC secretome, or hFFSC secretome $(5 \mathrm{ng} / \mathrm{ml})$ for $48 \mathrm{~h}$. Next, HSCs were incubated with $4 \%$ paraformaldehyde at room temperature for 10 minutes, and then incubated with $1 \%$ bovine serum albumin (BSA, Biosharp, Wuhan, China) for 30 minutes. Cells were incubated with a primary antibody against a-SMA (ab5694, 1:100 dilution, Abcam, Cambridge, UK) for 1h, followed by incubation with a secondary antibody (goat anti-rabbit IgG, ab15007, 1:500 dilution, Abcam, Cambridge, UK) for 30 minutes at room temperature. Rhodamine phalloidin (Thermal Scientific, Waltham, U.S.) was stained for cytoskeleton. The nuclei were labeled with DAPI (Thermal Scientific, Waltham, U.S.). Fluorescent images were captured using an EVOS Cell Imaging System (Thermo Scientific, Waltham, U.S.).

\section{Biochemical analysis}

To determine the liver function, we processed serum samples to measure following markers, the serum levels of alanine aminotransferase (ALT), aspartate aminotransferase (AST), total protein (TP), total bilirubin (TBIL), alkaline phosphatase (ALP), gamma-glutamyl transpeptidase ( $\mathrm{Y}-\mathrm{GT}$ ) were assessed using the Automated Biochemical Analyzer (AU-680, Beckman, California, U.S.) according to the procedure. Liver homogenate $(10 \%, \mathrm{w} / \mathrm{v})$ was prepared by homogenizing the right lobe of liver on ice in $150 \mathrm{mM}$ Tris$\mathrm{HCl}$ buffered saline (pH 7.2) using a polytron homogenizer (PT3100D; Kinematical, Lucerne, Switzerland). The levels of Malondialdehyde (MDA) and Hydroxyproline (Hyp) in liver tissue were measured using kits (Nanjing JianCheng Bio., Nanjing, China) according to the manufacturer's instructions. 


\section{Quantitative real-time PCR (qRT-PCR)}

HSCs were co-cultured with either PBS, hUCMSC secretome, or hFFSC secretome $(5 \mathrm{ng} / \mathrm{ml})$ for $48 \mathrm{~h}$ before samples were collected for mRNA extraction. Total RNA was isolated from HSCs using Trizol reagent (Invitrogen, Shanghai, China) according to the manufacturer's protocol. Then, $1 \mu \mathrm{g}$ total RNA was reversetranscribed to give CDNA, which was used as the template, and combined with standard SYBR premix Ex Taq (Invitrogen, Shanghai, China) on the Real-Time PCR Detection System (Roche, Basel, Switzerland), and experiments were conducted in triplicate. The levels of EMT related genes (E-cadherin, Snail1, Vimentin, FSP1 and a-SMA) , TGF- $\beta$ /Smad signaling pathway-related genes (TGF- $\beta 1$, Smad2, Smad3, Smad7 and Collagen I), and the internal standard GAPDH mRNA were measured by qRT-PCR. The primers are listed in Table S1, and GAPDH served as the internal control. All reactions were performed in triplicate and the data were analyzed using the $2^{-\Delta \Delta C t}$ method.

\section{Western blotting}

HSCs were co-cultured with either PBS, hUCMSC secretome, or hFSSC secretome $(5 \mu \mathrm{g} / \mathrm{ml})$ for $48 \mathrm{~h}$ before samples were collected for protein extraction. Liver tissue was collected from each treatment group for protein extraction. Protein samples were mixed with SDS sample lysis buffer and heated to $95^{\circ} \mathrm{C}$ for 10 minutes, followed by separation on SDS-polyacrylamide gels. Resolved proteins were electro-blotted onto nitrocellulose membrane and probed with antibodies against Ki-67(ab15580), HNF-4a(ab219610), TGF-

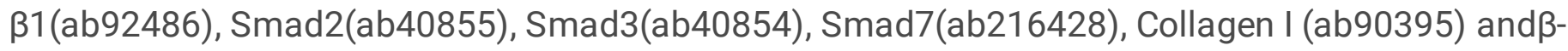
actin(ab5694), (1:1000 dilution, Abcam, Cambridge, UK) overnight at $4{ }^{\circ} \mathrm{C}(1: 1000$ dilution, Abcam, Cambridge, UK). Nitrocellulose membranes were then incubated with a secondary antibody, HRPconjugated goat anti-rabbit IgG (ab15007), at room temperature for $2 \mathrm{~h}$, and visualized by chemiluminescent detection according to the manufacturer's instructions (Immobilon western chemiluminescent HRP substrate, Millipore, Massachusetts, U.S.).

\section{Statistical analysis}

Statistical analysis was performed using GraphPad Prism Version 6. One-way ANOVA with post-hoc Dunnett's multiple comparisons test was used to test for statistically significant differences among the groups. All quantitative data are expressed as mean \pm SD. We performed at least three independent experiments, and $p<0.05$ was considered to be statistically significant.

\section{Results}

\section{In vivo: hFSSC secretome reduced $\mathrm{CCl}_{4}$-induced liver fibrosis in rats.}

To explore the therapeutic effect of hFSSC secretome on liver fibrosis, we used $\mathrm{CCl}_{4}$-induced a liver fibrosis rat model (Fig. 1a). The gross anatomical evaluation of the liver we observed distinguish morphology recovery in the hFSSC secretome treatment group, including less fibrous nodular, more ruddy on the surface, more uniform surface, and soft texture compared to the liver fibrosis treated with PBS 
(Fig. 1b). After 4 weeks treatment, histopathologic analysis using Masson and Sirius red staining which is an indicator of collagenase. We observed a significant reduction in the area of Collagen (in percentage) present in the liver treated with hFSSC group (9.2\%), compared to the other two control groups (24.3\% in PBS group and $14.9 \%$ in hUCMSC secretome group, Fig. $1 \mathrm{~b}$ and $1 \mathrm{c}, p<0.05)$. The Masson and Sirius red staining results indicate a significant reduction of the collagen area, which refers to the reduction in liver injury.

Furthermore, we measured the other liver marker such as, MDA (a marker for oxidative stress and liver cell injury) and Hyp (the main component in collagen tissue) content in the liver tissue samples. We found that the level of MDA and Hyp in the hFSSC secretome treatment group was significantly lower than the other two control groups (Fig. 1d and 1e $p<0.01$ ). These findings suggest that hFSSC secretome effectively reduced $\mathrm{CCl}_{4}$-induced liver fibrosis in rats.

\section{In vitro: hFSSC secretome reduced liver fibrosis through suppressing the EMT}

To further verify the roles of hFSSC secretome in the pathogenesis of liver fibrosis, we performed immunofluorescence staining of TGF- $\beta$ in HSCs. TGF- $\beta 1$ is considered as a crucial mediator in tissue fibrosis, and HSCs are one of the primary effector cells in liver fibrosis. In the current study, we found that hFSSC secretome group reduced fluorescence intensity observably, compared to the other two control groups (Fig.2a). In qRT-PCR studies, we explore the effect of hFSSC secretome on EMT, and examine the expression levels of EMT-related indicators (E-cadherin, Snail1, Vimentin, FSP1 and a-SMA in HSCs). Interestingly, our results found that hFSSC secretome treatment increased the epithelial marker of Ecadherin expression, while decreased the transcription factors of Snail 1 and mesenchymal markers (Vimentin, FSP1 and a-SMA) expression levels, compared to the PBS group (Fig. 2b, $p<0.05$ ). Meanwhile, we also examined hFSSC secretome increased E-cadherin and decreased FSP1 and a-SMA expression compared to the hUCMSC secretome group (Fig. $2 b, p<0.05$ ). These results suggest that hFSSC secretome reduced liver fibrosis through suppressing the EMT.

\section{In vivo: hFSSC secretome improved liver functionality and promoted liver regeneration}

To explore the effect of hFSSC secretome on liver functionality, we performed the biochemical analyses to measure the common liver functional markers. The results show that in comparison to the PBS group, hFSSC secretome group significantly reduced serum levels of ALT, AST, TBIL, $\mathrm{Y}$-GT, and ALP (Fig. 3a-3e, $p$ $<0.05)$. However, the serum level of TP in hFSSC secretome group was significantly higher than that in PBS group (Fig. $3 f, p<0.05$ ). In addition, hFSSC secretome group significantly reduced the serum levels of TBIL and $y-G T$ compared to hUCMSC secretome group (Fig. $3 c$ and $3 d, p<0.05$ ). These results suggest that hFSSC secretome effectively improved liver functionality.

Next, we performed IHC to assess the effects of the hFSSC secretome on the liver. a-SMA, which is an essential indicator of the occurrence and development of hepatic fibrosis. IHC results showed that the percentage of a-SMA positive area in hFSSC secretome group $(0.82 \%)$ was significantly decreased compared to the PBS group (5.51\%, Fig. $4 \mathrm{a}$ and $4 \mathrm{~d}, p<0.001)$. IHC results showed that the percentage of 
PCNA positive areas in hFSSC secretome group ( $4.13 \%$ ) was significantly increased compared to the PBS group (7.48\%, Fig. $4 a$ and 4 e, $p<0.01)$. The consist results correlated with the biochemical and qRTPCR results, the percentage of HNF-4apositive area was significantly increased in hFSSC secretome group $(12.1 \%)$, compared to the PBS group $(4.5 \%, p<0.01)$ as well as hUC-MSCs group ( $8.2 \%$, Fig. 4 a and $4 \mathrm{f}, p<0.05)$. Additionally, we performed an immunoblots to quantify the total amount proliferation by measuring Ki-67 and HNF-4a ratio levels in the livers lystate and with the consistent to the above immunocytochemistry results (Fig. 4b). We found that the ratio of Ki-67/ HNF-4a siginificantly increased in hFSSC secretome group, compared to the other two control group (Fig. 4c, $p<0.05$,). These results indicated that hFSSC secretome can effectively promote hepatocyte proliferation and cause liver regeneration, and furthermore preventing the progression of liver fibrosis in the rat model.

\section{In vivo: $\mathrm{hFSSC}$ secretome regulate the TGF- $\beta /$ Smad signal pathway}

To investigate the underlying mechanism of the effect of hFSSC secretome on liver fibrosis, we performed the Western blot and qRT-PCR to analyze the protein and gene expression of TGF- $\beta 1$, Smad2, Smad3, Smad7 and Collagen I in HSCs, as this markers are one of the primary effector cells in liver fibrosis. We found that TGF- $\beta 1$, Smad2, Smad3, and Collagen I expression was significantly decreased in hFSSC secretome group, compared to that of the PBS group (Fig. $5 a$ and $5 b, p<0.001$ ). However, we detected the Smad7 was significantly increased in hFSSC secretome group, compared to that of the other two control groups (Fig.5a and $5 b, p<0.01$ ). Smad7 serves as a negative feedback regulator of the TGF$\beta 1 /$ Smad pathway, thereby protects against TGF- $\beta 1$-mediated fibrosis (Fig. 5). These results suggest that hFSSC secretome effectively reduced liver fibrosis by regulating the TGF- $\beta /$ Smad signal pathway.

\section{Discussion}

In this study, our results demonstrated that hFSSC secretome reduces liver fibrosis in $\mathrm{CCL}_{4}$ induced fibrosis. Moreover, our research illustrated that hFSSC secretome reduces liver fibrosis through suppressing the EMT and regulating the TGF- $\beta$ /Smad signal pathway in HSCs.

Recent evidence indicates that the mesenchymal stem cell secretome as an acellular regenerative therapy for the liver disease[5, 14]. The use of MSC secretome has been shown to have anti-fibrotic effects[14]. Moreover, IV injection of hucMSC-EVs decreased liver fibrosis, reduced apoptosis, and mitigated liver damage induced by $\mathrm{CCl}_{4}$ in mice[15, 16]. In our study, we found that hFSSC secretome effectively reduced $\mathrm{CCl}_{4}$-induced liver fibrosis, and improved liver functionality in rats (Fig.1). Moreover, hFSSCs secretome treatment is more effective than of hUCMSCs secretome in resolving fibrosis, such as some indicators included collagen area, MDA, Hyp, TBIL, and $\mathrm{y}-\mathrm{GT}$. The reasons for the differences in the result between hUCMSCs, and hFSSCs secretome may be due to the difference in the presence more biological activity factors contain in hFSSCs secretome than hUCMSCs secretome[13]. Our studies indicated that hFSSC secretome is an attractive emerging option for therapeutic applications as a therapeutic strategy for liver fibrosis. 
Previous studies that mesenchymal stem cell secretome inhibits HSC activation and promoted liver regeneration[17-19]. HSCs stimulate the production of a large number of collagen fibers to form liver fibrosis. Moreover, positive expression of a-SMA can serve as a marker for HSCs activation[20]. Our results demonstrate that hFSSC secretome decreased a-SMA expression. PCNA and HNF-4ais two crucial indicator of hepatocyte proliferation[15]. Our study demonstrates that the ratio of Ki-67/ HNF-4awas significantly increased after hFSSC secretome treatment. These results confirmed that hFSSC secretome promotes liver regeneration while reducing the intensity of the liver fibrosis. Despite our results confirming that hFSSC secretome had significantly reduced liver fibrosis and caused no detectable immunological responses, their exact mechanisms of action need further exploration.

Moreover, the study has reported that type 2 EMT is associated with tissue regeneration and organ fibrosis. After $\mathrm{CCl}_{4}$ injury in liver, inflammatory cytokines, or chemical substances stimulate EMT to produce new collagen fibroblast pools to repair injury[5]. In the present study, we demonstrated that hUCMSC secretome reduced liver fibrosis through suppressing the EMT process.

The hucMSC secretome secreted some growth factors and cytokines, such as hepatocyte growth factor (HGF), transforming growth factor-beta isoform 3 (TGF- $\beta 3$ ), and tumor necrosis factor-alpha (TNF- $a$ ), and IL-10 can modulate cell signaling and processes involved in fibrogenesis and can attenuate liver fibrosis[5, 21]. Recently, fetal mesenchymal stem cells functional secretome analysis illustrates that $737 \pm 80$ protein identifications were obtained from the amniotic fluid-mesenchymal stem cells (AF-MSCs) secretome, interestingly, it reveals that Annexin-A1 as an important paracrine factor in hepatic regeneration[22]. However, the previous study demonstrated that hFSSC presented with the characteristics of both MSC and embryonic stem cell (ESC)[13]. These may be the partial reason that hFSSC secretome can effectively reduce liver fibrosis, and has better effects than hUCMSC secretome in some indicators levels.

The TGF- $\beta 1 /$ Smad pathway is an essential pathogenic mechanism in tissue fibrosis[23, 24]. Studies have shown that TGF- $\beta 1$ is considered as a crucial mediator in tissue fibrosis and causes tissue scarring mainly by activating its small downstream molecules against decapentaplegic (Smad) signaling[25]. However, different TGF- $\beta$ signaling play different important roles in fibrogenesis[24]. TGF- $\beta 1$ directly activates Smad signaling, which triggers cause the pro-fibrotic gene to overexpress[26]. Increasing studies have demonstrated that dysregulation of TGF- $\beta 1 /$ Smad pathway was an important pathogenic mechanism in tissue fibrosis[27]. Smad2 and Smad3 are the two major downstream regulators that promote TGF- $\beta 1$-mediated tissue fibrosis, while Smad7 serves as a negative feedback regulator of the TGF- $\beta 1 /$ Smad pathway thereby protects against TGF- $\beta 1$-mediated fibrosis[24]. Our findings proved that hFSSC secretome effectively reduced TGF- $\beta 1$, Smad2, Smad3, and Collagen I expression, moreover, increased Smad7 expression. It indicated that hFSSC secretome effectively reduced liver fibrosis via regulating the TGF- $\beta /$ Smad signal pathway (Fig. 6).

In conclusion, we successfully investigated the role of hFSSC secretome on cutaneous liver fibrosis. Our results demonstrated that hFSSC secretome could exert the protective effect of liver fibrosis via 
regulating the TGF- $\beta /$ Smad signal pathway.

\section{Declarations}

\section{Ethics approval and consent to participate}

All the protocols and procedures were approved by the Animal Experiment Ethics Committee of the Changchun University of Traditional Chinese Medicine, China (Approval NO. XW201903167). The informed written consent form was approved by the Clinical Research Ethics Committee and signed by the donor before sample collection.

\section{Consent for publication}

Not applicable.

\section{Availability of supporting data}

The datasets used and/or analyzed during the present study are available from the corresponding author on reasonable request.

\section{Competing interests}

The author(s) declared no potential conflicts of interest with respect to the research, authorship, and/or publication of this article.

\section{Funding}

This work was supported by the National Natural Science Foundation of China (No.8762069).

\section{Authors' Contributions}

X.Y. carried out the cell culture and animal studies, and helped to draft the manuscript. J.W. carried out animal studies and qRT-PCR. J.Z. carried out WB and performed the statistical analysis. X.R. conceived of the study, and participated in its design and coordination and helped to draft the manuscript.

\section{Acknowledgments}

The authors thank Professor Chen, Changchun University of Traditional Chinese Medicine, for his assistance in animal experiment.

\section{Abbreviations}

hFSSC: Human fetal skin-derived stem cell; MSCs: mesenchymal stem cells; hUCMSCs: human umbilical cord mesenchymal stem cell; HSCs: Hepatic stellate cells; ALT: Alanine aminotransferase; AST: Aspartate aminotransferase; TBIL: Total bilirubin; ү- GT: Gamma glutamyl transpeptidase; ALP: Alkaline 
phosphatase; TP: Total protein; Hyp: Hydroxyproline; MDA: Malonaldehyde; qRT-PCR: Quantitative realtime PCR; a-SMA: Alpha-smooth muscle actin; HGF: Hepatocyte growth factor; EMT: Epithelial to mesenchymal transition; TNF-a:Tumor necrosis factor-alpha; ESC: Embryonic stem cell; FSP1: fibroblastspecific protein 1; AF-MSCs: Fluid-mesenchymal stem cells; ESC: Embryonic stem cell

\section{References}

1. Sun M, Kisseleva T: Reversibility of liver fibrosis. Clinics and research in hepatology and gastroenterology 2015, 39 Suppl 1:S60-63.

2. Arriazu E, Ruiz de Galarreta M, Cubero FJ, Varela-Rey M, Perez de Obanos MP, Leung TM, Lopategi A, Benedicto A, Abraham-Enachescu I, Nieto N: Extracellular matrix and liver disease. Antioxid Redox Signal 2014, 21(7):1078-1097.

3. Elpek GO: Cellular and molecular mechanisms in the pathogenesis of liver fibrosis: An update. World J Gastroenterol 2014, 20(23):7260-7276.

4. Konala VB, Mamidi MK, Bhonde R, Das AK, Pochampally R, Pal R: The current landscape of the mesenchymal stromal cell secretome: A new paradigm for cell-free regeneration. Cytotherapy 2016, 18(1):13-24.

5. Driscoll J, Patel T: The mesenchymal stem cell secretome as an acellular regenerative therapy for liver disease. Journal of gastroenterology 2019, 54(9):763-773.

6. Rong X, Liu J, Yao X, Jiang T, Wang Y, Xie F: Human bone marrow mesenchymal stem cells-derived exosomes alleviate liver fibrosis through the Wnt/beta-catenin pathway. Stem Cell Res Ther 2019, 10(1):98.

7. Moerkamp AT, Lodder K, van Herwaarden T, Dronkers E, Dingenouts CK, Tengstrom FC, van Brakel TJ, Goumans MJ, Smits AM: Human fetal and adult epicardial-derived cells: a novel model to study their activation. Stem Cell Res Ther 2016, 7(1):174.

8. Ishii T, Eto K: Fetal stem cell transplantation: Past, present, and future. World J Stem Cells 2014, 6(4):404-420.

9. Zhang ZY, Teoh SH, Hui JH, Fisk NM, Choolani M, Chan JK: The potential of human fetal mesenchymal stem cells for off-the-shelf bone tissue engineering application. Biomaterials 2012, 33(9):2656-2672.

10. Xu J, Wang B, Sun Y, Wu T, Liu Y, Zhang J, Lee WY, Pan X, Chai Y, Li G: Human fetal mesenchymal stem cell secretome enhances bone consolidation in distraction osteogenesis. Stem Cell Res Ther 2016, 7(1):134.

11. Chan J, O'Donoghue K, de la Fuente J, Roberts IA, Kumar S, Morgan JE, Fisk NM: Human fetal mesenchymal stem cells as vehicles for gene delivery. Stem cells (Dayton, Ohio) 2005, 23(1):93-102.

12. Wang X, Jiao Y, Pan Y, Zhang L, Gong H, Qi Y, Wang M, Gong H, Shao M, Wang X et al: Fetal Dermal Mesenchymal Stem Cell-Derived Exosomes Accelerate Cutaneous Wound Healing by Activating Notch Signaling. Stem Cells Int 2019, 2019:2402916. 
13. Rong X, Li J, Yang Y, Shi L, Jiang T: Human fetal skin-derived stem cell secretome enhances radiation-induced skin injury therapeutic effects by promoting angiogenesis. Stem Cell Res Ther 2019, 10(1):383.

14. Jang YJ, An SY, Kim JH: Identification of MFGE8 in mesenchymal stem cell secretome as an antifibrotic factor in liver fibrosis. BMB reports 2017, 50(2):58-59.

15. Li T, Yan Y, Wang B, Qian H, Zhang X, Shen L, Wang M, Zhou Y, Zhu W, Li W et al: Exosomes derived from human umbilical cord mesenchymal stem cells alleviate liver fibrosis. Stem cells and development 2013, 22(6):845-854.

16. Lou G, Chen Z, Zheng M, Liu Y: Mesenchymal stem cell-derived exosomes as a new therapeutic strategy for liver diseases. Exp Mol Med 2017, 49(6):e346.

17. Li Q, Zhou X, Shi Y, Li J, Zheng L, Cui L, Zhang J, Wang L, Han Z, Han Y et al: In vivo tracking and comparison of the therapeutic effects of MSCs and HSCs for liver injury. PLoS One 2013, 8(4):e62363.

18. Berardis S, Dwisthi Sattwika P, Najimi M, Sokal EM: Use of mesenchymal stem cells to treat liver fibrosis: current situation and future prospects. World J Gastroenterol 2015, 21(3):742-758.

19. Eom YW, Shim KY, Baik SK: Mesenchymal stem cell therapy for liver fibrosis. Korean J Intern Med 2015, 30(5):580-589.

20. Bataller R, Brenner DA: Liver fibrosis. Journal of Clinical Investigation 2005, 115(2):209-218.

21. Alfaifi M, Eom YW, Newsome PN, Baik SK: Mesenchymal stromal cell therapy for liver diseases. $J$ Hepatol 2018, 68(6):1272-1285.

22. Zagoura D, Trohatou O, Makridakis M, Kollia A, Kokla N, Mokou M, Psaraki A, Eliopoulos AG, Vlahou A, Roubelakis MG: Functional secretome analysis reveals Annexin-A1 as important paracrine factor derived from fetal mesenchymal stem cells in hepatic regeneration. EBioMedicine 2019, 45:542-552.

23. Hu HH, Chen DQ, Wang YN, Feng YL, Cao G, Vaziri ND, Zhao YY: New insights into TGF-beta/Smad signaling in tissue fibrosis. Chemico-biological interactions 2018, 292:76-83.

24. Walton KL, Johnson KE, Harrison CA: Targeting TGF-beta Mediated SMAD Signaling for the Prevention of Fibrosis. Frontiers in pharmacology 2017, 8:461.

25. Mokoena D, Dhilip Kumar SS, Houreld NN, Abrahamse H: Role of photobiomodulation on the activation of the Smad pathway via TGF-beta in wound healing. Journal of photochemistry and photobiology B, Biology 2018, 189:138-144.

26. Grotendorst GR: Connective tissue growth factor: a mediator of TGF-beta action on fibroblasts. Cytokine \& growth factor reviews 1997, 8(3):171-179.

27. Lindert S, Wickert L, Sawitza I, Wiercinska E, Gressner AM, Dooley S, Breitkopf K: Transdifferentiationdependent expression of alpha-SMA in hepatic stellate cells does not involve TGF-beta pathways leading to coinduction of collagen type I and thrombospondin-2. Matrix Bio/ 2005, 24(3):198-207.

\section{Figures}




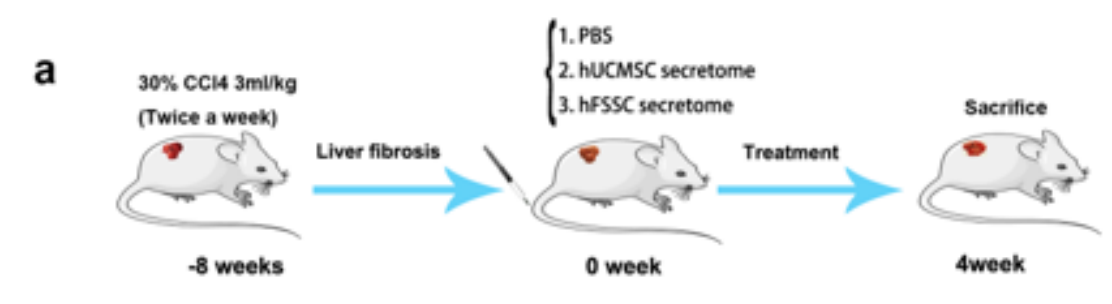

b

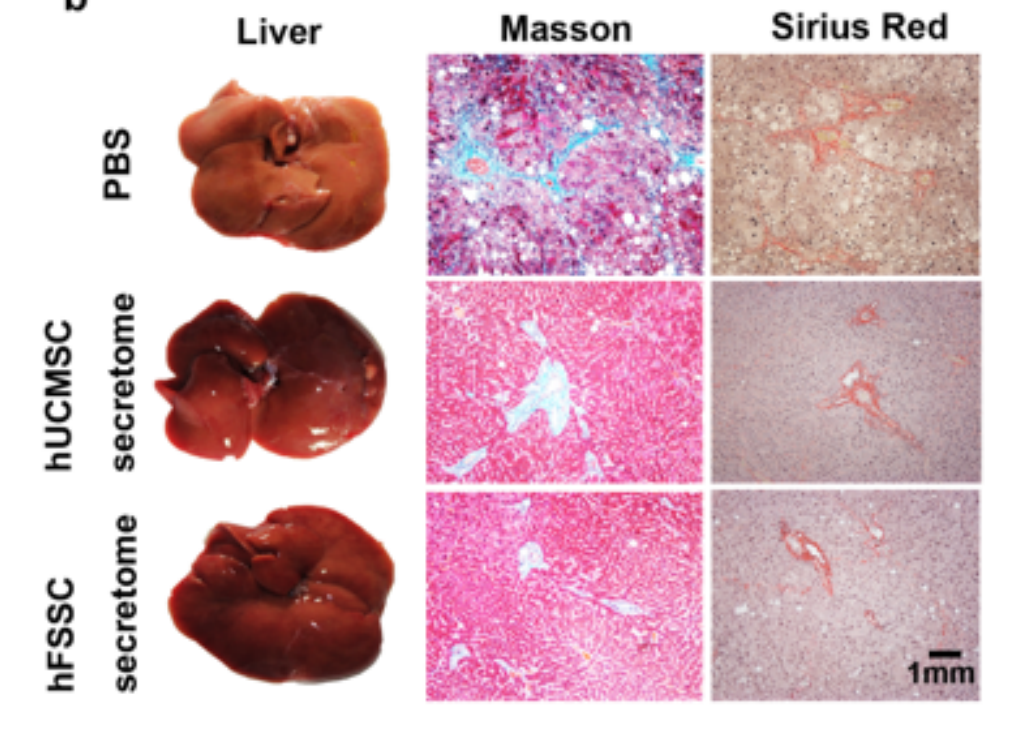

C

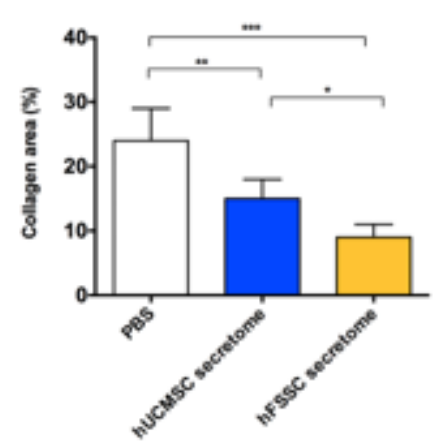

d

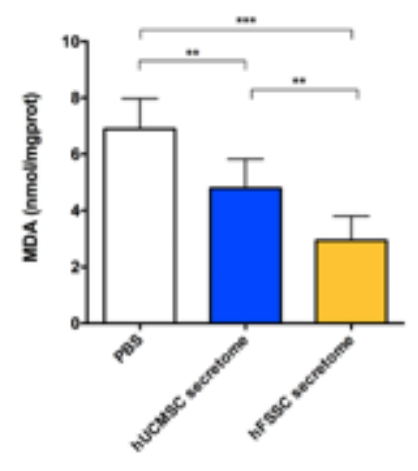

e

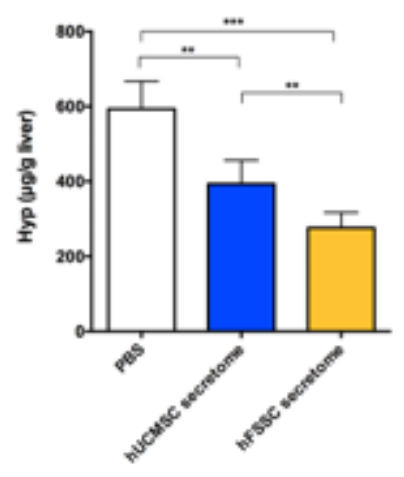

Figure 1

hFSSC secretome reduced liver fibrosis in rats. a. Experimental design and experiment performed in the illustration. b. The representative images of gross liver morphology (left panel), The representative images from Masson, and Sirius red staining of liver (middle and right panel). Bar $=1 \mathrm{~mm}, \mathrm{n}=10$. c. Quantitative image analysis of the collagen area in percentage at four weeks in different treatment groups. d. Quantitative analysis of hepatic MDA and e. Hyp content liver sections. ${ }^{*} p<0.05,{ }^{\star \star} p<0.01$, ${ }^{\star * \star} p<0.001 . n=10$; mean \pm SD. 

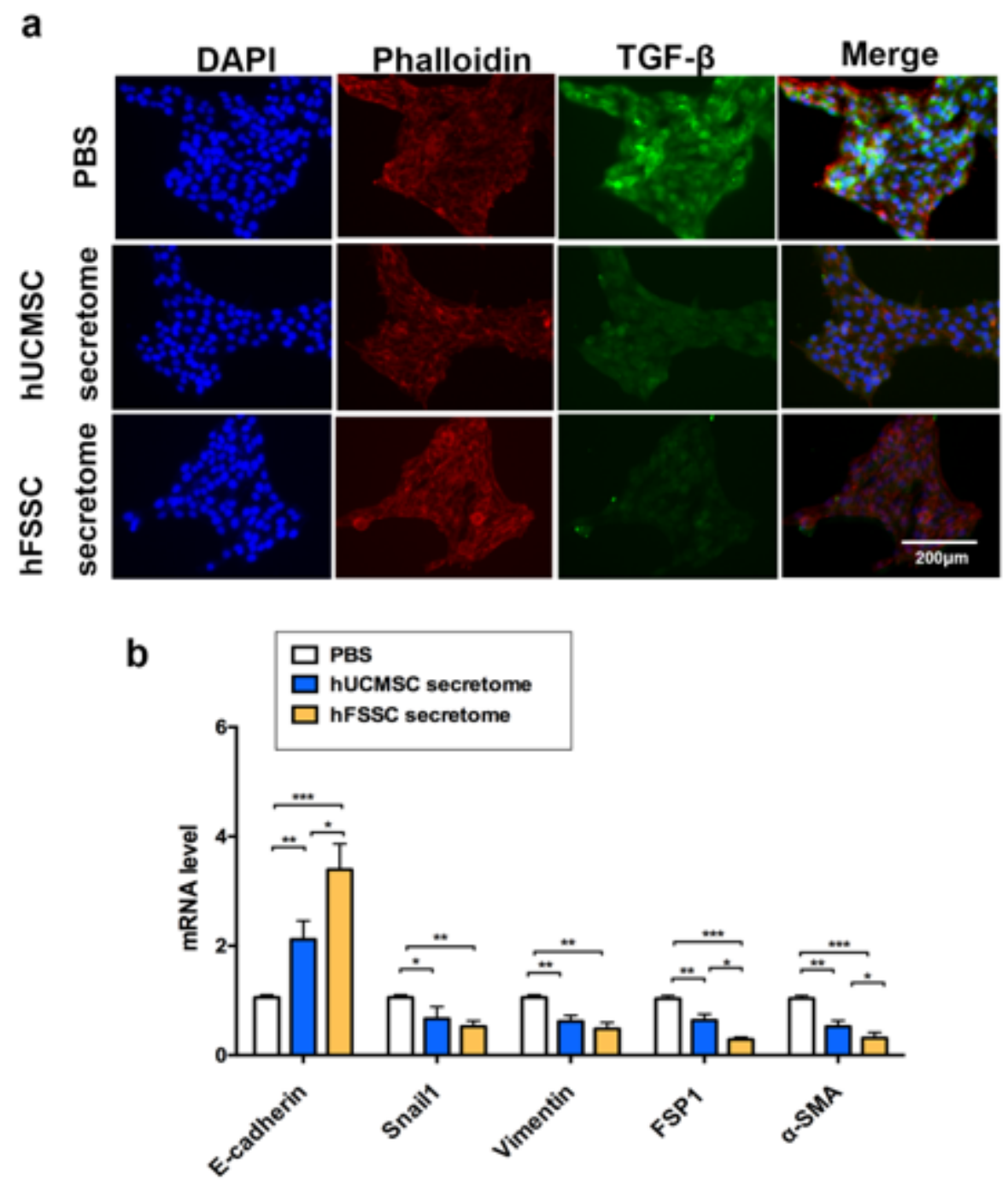

Figure 2

In vitro: hFSSC secretome reduced liver fibrosis through suppressing the EMT process. a. Representative images of immunofluorescence staining performed for TGF- $\beta$ in HSCs. Bar $=200 \mu \mathrm{m}$. b. Relative mRNA expression levels of EMT related gene, included of E-cadherin, Snail1, Vimentin, FSP1, and a-SMA in HSCs. Note that EMT: epithelial-mesenchymal transition; FSP1: fibroblast-specific protein 1; a-SMA: alpha-smooth muscle actin. ${ }^{*} p<0.05,{ }^{* \star} p<0.001,{ }^{* \star *} p<0.0001 . n=10$, mean $\pm S D$. 

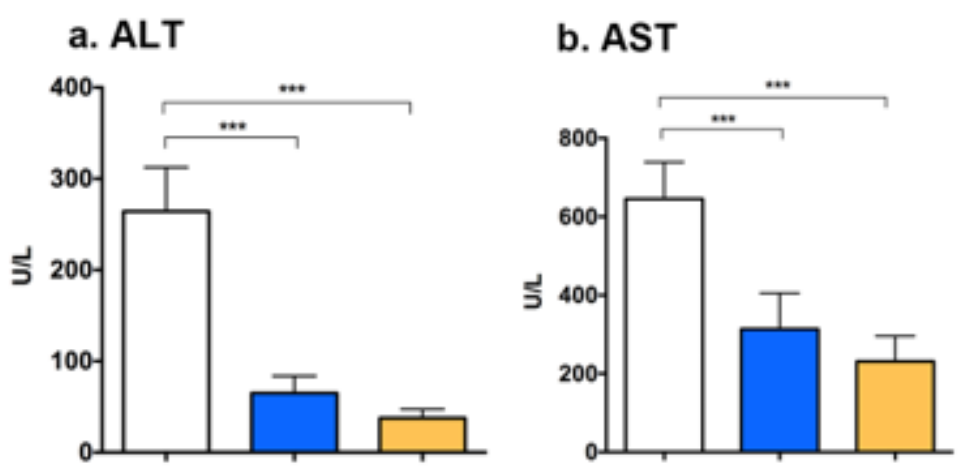

c. TBIL

\section{d. Y-GT}

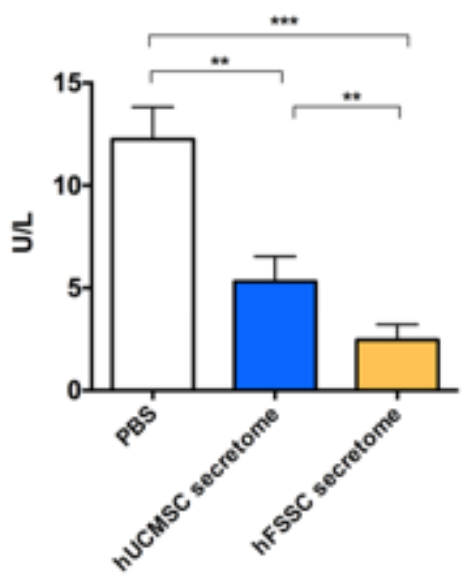

e. ALP

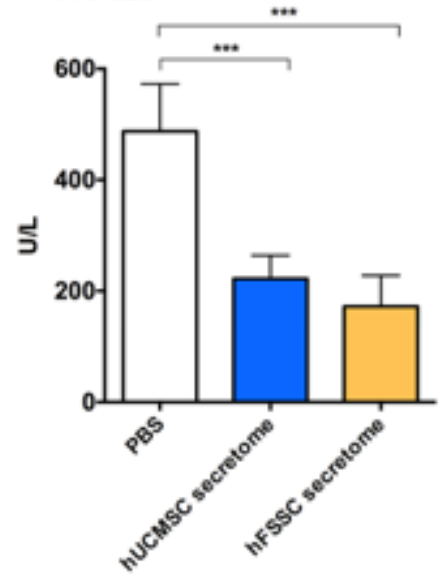

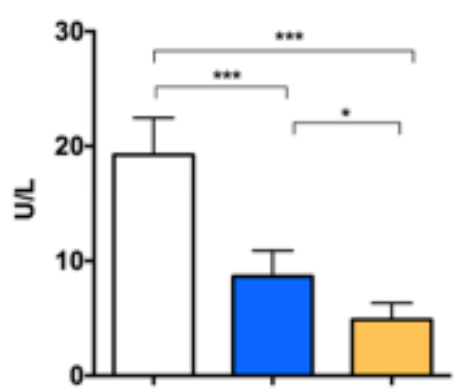

f. TP

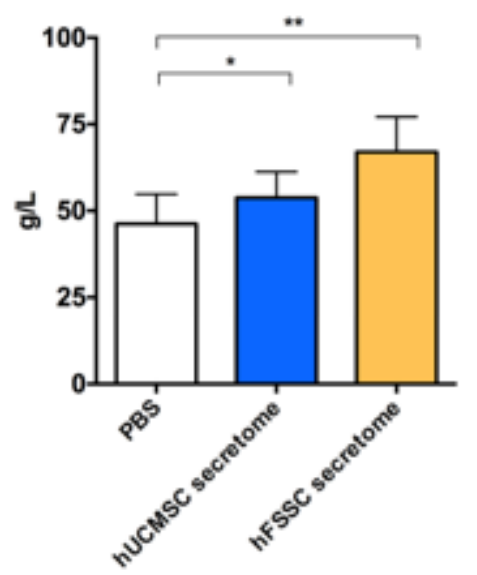

Figure 3

hFSSC secretome improves liver function in serum biochemical parameters. Serum samples were processed and analyzed for liver functional assay in the different treatment groups. a. ALT: Alanine aminotransferase. b. AST: Aspartate aminotransferase. c. TBIL: Total bilirubin. d. y-GT: Gamma-glutamyl transpeptidase. e. ALP: Alkaline phosphatase. f. TP: Total protein. ${ }^{*} p<0.05,{ }^{\star \star} p<0.01,{ }^{\star \star \star}{ }^{*}<<0.001 . n=10$, mean \pm SD. 
a

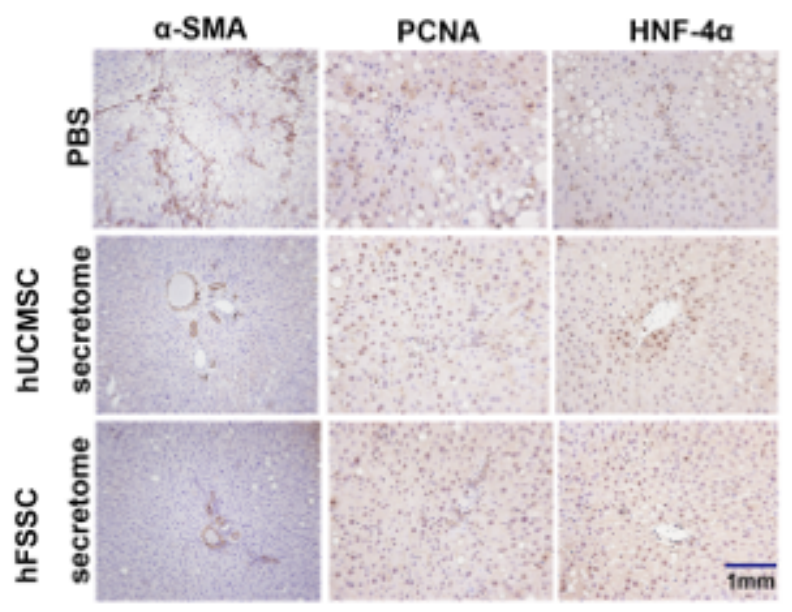

b

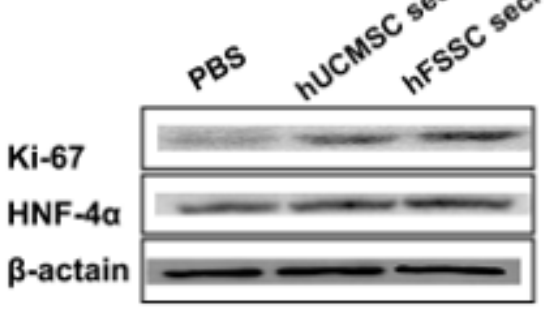

c

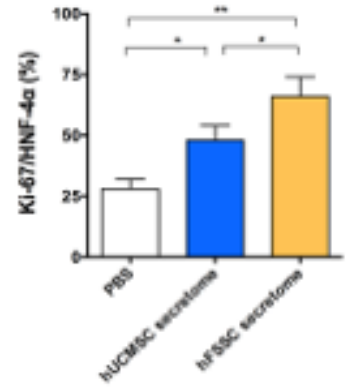

f

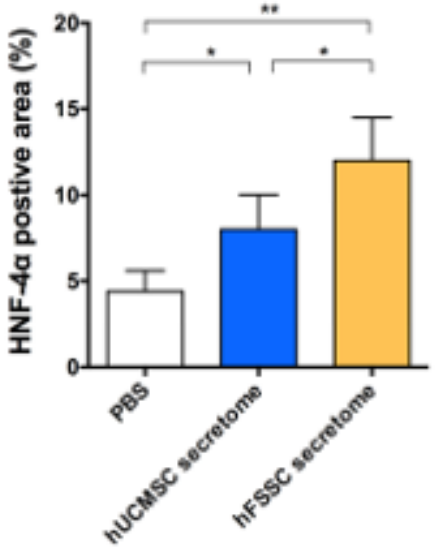

Figure 4

Histological analysis of hFSSC secretome reduced liver fibrosis and promoted liver regeneration. a. Photomicrographs shows liver sections staining for a-SMA, PCNA, and HNF-4a. DAB (Brown) stained cells represent the positive expression of representative markers for a-SMA, PCNA, and HNF-4a, bar = $1 \mathrm{~mm}$. b Representative immunoblots show protein expression of Ki-67 and HNF-4a in live tissue. c Qualitative analysis the ratio of Ki-67/HNF-4a in immunoblots. d-f. The quantification of a-SMA, PCNA, and HNF-4a positive cells area. ${ }^{\star} p<0.05,{ }^{* \star} p<0.01,{ }^{\star \star *} p<0.001 . n=10$, mean \pm SD. 

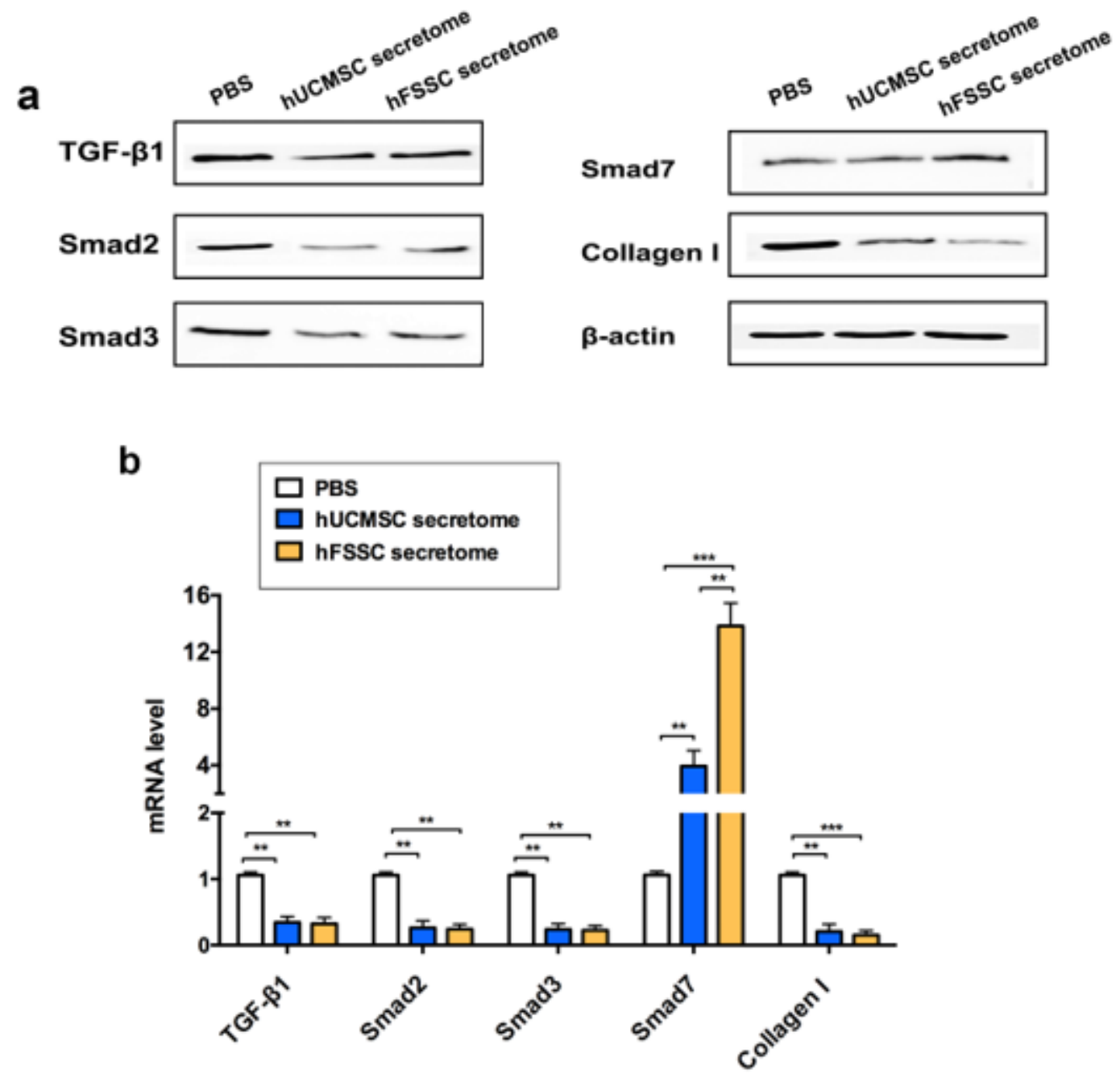

Figure 5

hFSSC secretome inhibited the TGF- $\beta$ /Smad signaling pathway in HSCs. a. Representative immunoblots show protein expression of TGF- $\beta 1$, Smad2, Smad3, Smad7, and Collagen I in HSCs. b. Qualitative analysis shows a relative mRNA expression levels of TGF- $\beta 1$, Smad2, Smad3, Smad7, and Collagen I in HSCs. ${ }^{* *} \mathrm{p}<0.001,{ }^{* *} \mathrm{p}<0.001 . \mathrm{n}=10$, mean \pm SD.

\section{Supplementary Files}

This is a list of supplementary files associated with this preprint. Click to download.

- SupplementaryMaterial.docx 